conds to be again occulted and tinally re-appeared in fifty seconds more. As the vecultation is an interesting one I send you the times precisely.

\begin{tabular}{|c|c|}
\hline Sid. Time Santiago. & Sid. Time Snntingo. \\
\hline 1st. In. $\quad 6^{1 / 41^{n 1} 54^{s} 7}$ & 2nd. $1 \mathrm{~m} .6^{\mathrm{h}} 45^{\mathrm{\prime \prime}} 8^{\mathrm{*}} 0$ \\
\hline 1st. Em. $644 \quad 31.1$ & $646 \quad 0,5$ \\
\hline
\end{tabular}

The Longitude we have last used is $4^{\mathrm{h}} 42^{\mathrm{m}} 18^{\mathrm{g}} 9$ and Latitude $33^{n} 26^{\prime} 24^{\prime \prime} 8$.

You will perceive that to those a third of a degree, indeed less than half that distance farther to the southward, the star nust have been passed by the moon without occultation, and yet the Nautical Almanac puts down the liniting parallels $6^{\circ} \mathrm{N}$. and $54^{\circ} \mathrm{S}$. or nearly a central occultation in our latitude. Nor was $\mu$ occulted at all, though marked as possibly visible to those 750 miles south of us. Governed by the Almanac, I have lost almost as many hours in going to the ohservatory to olserve stars which were not occulted as it would have taken to calculate them before hand. For several I have gone up Sinta Lucia at very advanced hours of the night, only to be disappointed, as for instance Mars on the 14th Nov. near 3 A. M.

The differential ohservations with this planet and stars near its path, were commenced in accordance with the ephemeris of which copies were distrihuted. Since then (15th Dec.) there have been 35 uights, out of which there have been observations with both the equatoreal and neridian circle on 27 with the meridian only 31 . In December the number of differentials was 365 , the 15 th, 17 th, 18 th and 23 ud. being cloudy. Many of my measures lave given ne great satisfaction, and if observers in the ourthern henisphere have been equally fortunate, we shall have a right to expect an interestiug result.

\title{
Note sur la manière de calculer le décroissement d'intensité que la Photosphère du Soleil subit en traversant l'atmosphère qui l'entoure, par Jean Plana.
}

1.

L'opinion, que la photosphère du Soleil est entourée d'une couche gazeuse, capable d'affaiblir l'intensité da sa lumière, paraît anjourd'bui généralement admise. On explinue par là l'expérience de Bouguer sur le décroissement d'intensité de la lumière du Soleil depuis le centre vers ses hords. La surface rayounante du Soleil étant sphériquement enceloppée par une couche gazeuse absorbante, il doit arriver, que l'affaiblissement de la lumière sera d'autant plus grand, que l'épaisseur de la couche qu'elle traverse est plus considérable. Cette manière de vnir est conlirmée par la mesure des actions photogéosiques, et des actions calorifiques, qui accompagnent la lumière du Soleil. A ces faits il faut ajouter celui des teintes rosacées, séparées du limbe de la Lune, teintes qui furent observées pendant les éclipses totales du Soleil du 8 aont 1850 et du 28 juillet 1851 . Car ces teintes peurent ètre assinilées (comme le dit fort bien Mr. Arago) „à des nuages fluttants dans une atmosphère solaire douée d'un mouvement de rotation peu rapide."

Lapluce, dans le Tome 4. de sa Mécanique Céleste, (voyez page 282-288) a donné une formule pour calculer le décroissement d'iutensité de la lumière du Soleil qui serait du à la cause dont nous venons de parler; et en parlant du résultat d'une expérience de Bouguer, exposée à la page 91 de son Traité Sur la gradation de la lumière, il en a tiré la conséquence, "que le Soleil dépouillé de son atmos- phère nous paraîtrait douze fois plus lumineuse." Mais le rapport $\frac{35}{4} \frac{5}{x}$ de Bouguer, qui conduit à cette conséquence, étant, probablement, affecté d'une erreur, $j$ 'ai repris l'analyse de Laplace, afin de la présenter sous une forme, susceptible de faire connaître linlluence d'une correction qu'il serait nécessaire d'introduire dans le coefficient $\frac{35}{48}$ de Bouguer, d'après des expériences plus précises. Et si l'on veut, que ce coefficient soit exact, on verri que le décroissement d'intensité, consiléré par Laplace, doit ètre peu différent d'un douzième. En supposant que le nombre ${ }_{43}^{3.5}$ doive átre remplacé par $35+\frac{1}{43}$, lon aurait à-peu-près un dixième pour le dé croissement de l'intensité: mais, sil fallait le remplacer par $3 \frac{35}{4}-2^{1}$, le décroissement serait exprimé par la fraction T⿱亠䒑十.

2.

Soit $\mu=\frac{35}{4}$ : suivant les dénoninations de Laplace, on doit déterniner l'exposant $f$ d'après l'équation

$$
\frac{-f}{e^{\cos \theta}}=\mu \operatorname{ros} \theta \cdot e^{-f}
$$

qui donne (en employant les logarithmes hyperboliques)

$$
f=-\frac{\cos \theta}{2 \sin ^{2} \frac{1}{2} \theta}\{\text { Log. } \cdot n \cdot \theta+\log \cdot \mu\} \ldots(1)
$$

En prenant $\sin \theta=\frac{3}{4}$, on obtient

$$
f=1,42460 ; \quad e^{-f}=0,2406045
$$


Log. tab. $f=0,1536928$; Lng. hyp. $f=0,353890$. Cela posé; si $\mu+\delta \mu$ est la véritable valeur du coeflicient $\mu$, et $f+\delta f$ la véritable valeur correspundante de $f$, nous aurons

$$
\delta f=-\frac{\cos \theta}{2 \sin ^{2} \frac{1}{2} \theta} \cdot \frac{\delta \mu}{\mu}
$$

d'où l'on tire, dans le cas de ros $\theta=\frac{3}{4}$;

$$
\delta f=-2,67932 . \delta \mu ; \delta . e-f=0,644656 . \delta \mu .
$$

En prenant pour unité l'intensité de la lumière du Soleil dépouillé de l'atmosphère qui entuure sa photosphère, l'on a

$$
\frac{2 \pi \int_{0}^{\frac{\pi}{2}} d \theta \sin \theta \cdot \theta^{\frac{-f}{\operatorname{mos} \theta}}}{2 \pi \int_{0}^{\frac{\pi}{2}} d \theta \sin \theta}=\int_{0}^{\frac{\pi}{2}} d \theta \sin \theta \cdot \theta^{\frac{-f}{\cos \theta}}
$$

pour expression de l'intensité après le passage de la lumière solaire par la couche gazeuse. Donc, en posant $\cos \theta=x$, et nommant $I$ cette intensité, nous avons

$$
I=\int_{0}^{1} d x \cdot e^{-\frac{f}{x}} \ldots \ldots \ldots \ldots
$$

En intégrant par parties il est clair que l'on a

$$
I=e^{-f}-f \int_{0}^{1} \frac{d x}{x} e^{-\frac{f}{x}}
$$

Soit $x=f u$, et $u^{\prime}=\frac{1}{f}$, nous aurons

$$
I=e^{-f}-f \int_{0}^{u^{\prime}} \frac{d u}{u} e^{-\frac{1}{u}}
$$

Maintenant, si lon fait $u=\frac{1}{z}$, il viendra

$$
I=e^{-f}+f \int_{\infty}^{f} \frac{d z}{z} \cdot-f .
$$

En développlant cette intégrale détinie par la série posée à la page 505 du second Volume du Traité des fonctions elliptiques de Legendre, lion a

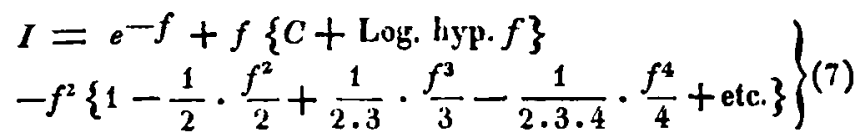

où $C=0,5772156649 \ldots$ En prenant $f=1,42460$ l'ou a $C+$ Log. hyp. $f=0,931105 ; f\{C+$ Log. hyp. $f\}$ $=1,326440$. La série étant calculée jusqu'au terme multiplié par $f^{8}$ inclusivement, on trouve

$$
\begin{aligned}
& I=0,2406045+1,326440-2,02947+0,722800 \\
& \quad-0,228823+0,061121-0,013931+0,00027 \mathrm{~s} 6 \\
& \quad-0,000042=2,3512411-2,272266=0,0789751 ;
\end{aligned}
$$

c'est - à - dire, $I=\frac{1}{12,662}$.

Pour faire ce calcul d'une manière plus expéditive, on pourrait observer que, en posant $y=e^{-2}$, et $y^{\prime}=e^{-f \text {, }}$ l'on $a$, au lieu de l'équation (6);

$$
I=e^{-f}+f \int_{0}^{y^{\prime}} \frac{d y}{\log \cdot y} \cdots \ldots \ldots
$$

Actuellement si l'on fait

$$
\nu=\int_{0}^{y^{\prime}} d y\left\{\frac{1}{1-y}+\frac{1}{\log \cdot y}\right\}
$$

l'on a

$$
I=e^{--f}+f\left\{V+\text { Log. hyp. }\left(1-y^{\prime}\right)\right\} \ldots \text { (10) }
$$

Avec cette formule, le calcul de l'intensité $I$ peut ètre exécuté immédiatement par la Table de Legendre (voyez page 510 du second Volume du Traité des fonctions elliptiques). Cette Table pour $y^{\prime}=0,2406045$ donne (en négligeant les secondes différences) $V=0,16330586$. Mais

Log. hyp. $\left(1-y^{\prime}\right)=-0,275340$; partant

$$
I=0,2406045-0,159605=0,080999=\frac{1}{12,31} .
$$

Soit $\psi(f)=\int_{0}^{1} d x \cdot e^{-\frac{f}{x}}:$ d'après le théorème de Leib. nitz, l'on a

$$
\frac{d \psi(f)}{d f}=-\int_{0}^{1} \frac{d x}{x} \cdot e^{-\frac{f}{x}} .
$$

Donc, en négligeant le carré de $\delta f$, nous avons, par le changement de $f$ en $f+\delta f$;

$$
I=\psi(f)-\delta f \int_{0}^{1} \frac{d x}{x} \cdot e^{-\frac{f}{x}}
$$

ou bien

$$
I=0,0789751-\delta f \int_{0}^{1} \frac{d x}{x} \cdot e^{-\frac{f}{x}} .
$$


Mais l'équation (4) donne

$$
-f \int_{0}^{1} \frac{d x}{x} \cdot e^{-\frac{f}{x}}=-e^{1}+\int_{0}^{1} d x \cdot e^{-\frac{f}{x}}
$$

donc, en substituant il viendra

$$
\begin{aligned}
& I=0,0789751-\frac{d f}{f}\{0,2406045-0,0789751\} \\
& I=0,0789751-\frac{\delta f}{f} \cdot 0,1616294
\end{aligned}
$$

et comme $\delta f=-2,67932 . \delta \mu$, notus avons

$$
I=0,0789751+0,30398 . \delta \mu \ldots \ldots \ldots(11)
$$

On voit par cette formule, que, en prenant $\delta \mu=\eta^{\prime} \delta$. la valeur de I devient à-peu-près un dixième; et que, en prenant $\delta \mu=-I^{\prime}$ elle s'abaisse $a$ un vingtiène. Cela suffit pour prouver que l'influence d'une erreur sur le coefficient $\mu$ $=\frac{35}{4}$ de Bouguer, peut être capable d'apporter une grande moditication sur la mesure de l'intensité $I$, qui reste à la photosphère du Soleil, après avoir termiıé l'atmosphère absorbante qui l'entoure.

Turin le 28 mai 1852 .

\section{Jean Plana.}

\section{Ueber die Säcularänderungen der Präcession im British Assoc. Catalogue.}

Ich erlaube mir hier eine kleine Bemerkung, die Berechnung der Säcularänderungen der Präcession betreffend. Sind $p$ und $p^{\prime}$ die einjăhrigen Prïcessionen in Rectascension und Declination, so ist bekanntlich $p=m+n \operatorname{tg} \delta \sin \alpha, p^{\prime}=n \cos \alpha$. Die Werthe $\alpha, \delta, m$ und $n$ gelten aber nur für eine bestimmte Epoche, nit ihnen ändern sich auch $p$ und $p^{\prime}$ and zwar il einem Jahre um

$$
\begin{aligned}
& d p=d m+d n \operatorname{tg} \delta \sin \alpha+n \operatorname{tg} \delta \cos \alpha \sin 1^{\prime \prime} d \alpha \\
& +n \sin \alpha \sec \delta^{2} \cdot \sin 1^{\prime \prime} d \delta, \\
& d p^{\prime}=d n \ln \alpha-n \sin \alpha \sin 1^{\prime \prime} d \alpha,
\end{aligned}
$$

weun $d m, d n$, $1 x$ und d die einjährigen Aenderungen jener Grössen bedeuten. Aber $d \alpha=p$, d $\delta=p^{\prime}$, und damn

$$
d p=d m+d n \operatorname{tg} \delta \sin \alpha+p p^{\prime} \operatorname{tg} \delta \sin 1^{\prime \prime}+p^{\prime 2} \sec d^{2} \operatorname{tg} \alpha \sin 1^{\prime \prime}
$$$$
d p^{\prime}=d n \cos \alpha-n p \sin x \sin 1^{\prime \prime} \text {. }
$$

Die Werthe derjenigen Glieder unn, welche $d m$ und $d n$ enthalten, sind häutig für so gering gehalten, dass sie ganz vernachlässigt werden können, oder es sind geradezu $d m$ und $d n=0$ gesetzt, während maı doch bei der gewöhnlichen Mathode der Reduction mit Hülfe dè für die Mitte da.r heiden Zeiten geltenden Präcession, auf die Aenderung von in und $n$ sehr wohl Rücksicht nimmt. Die Voraussetzung $d m$ und $d n$ $=0$ ist z. B. bei der Berechnung der Säcularänderungen in dem British Assoc. Cat. gemacht (Preface, p. 39). Sie sind daselbst bis auf 4 Stellen in AR.. Zeit. und 3 Stellen in Decl. angesetzt und daber die Vernachlässigung der erwähnten Glieder nur dann gestattet, wenn ihr Betrag den Werth einer Einheit dieser Stellen nicht erreicht. Es ist nun aber nach Bessel

$$
\begin{aligned}
\text { I's } 100 d_{m} & =+0,0020576, \quad \text { 1' } 100 d_{n}=-0,0006468, \\
100 d n & =-0,00970204
\end{aligned}
$$

und danit der Betrag, welcher an die Angalıen des Katalngs noch anzubringen ist $=+0,0020576-0,0006468 \operatorname{tg} \delta \sin \alpha$ in AR. und +0,00970204 ros $\alpha$ in Nordpolardistanz. Die fulgende erste: Tafel giebt nit den Argumenten $\alpha$ und $\delta$ den Werth -0,0006468 $\lg \delta \sin \alpha$, der dan!n noch mit dem richtigen Zeichen zu $+0,00206$ hinzuzufügen ist. Die Zeichen gelten für nördliche und sürliche Declinationen und für die ersten 12 Stunden der AR., sind also für $12^{\mathrm{h}}$ bis $23^{\mathrm{h}}$ die entgegengesetzten. Die Zahlen bedeuten Einheiteu der jten Stelle. Die zweite Tafel giebt mit dem Argument $\alpha$ die Correction für die Nordpolardistanz in Eimheiten der 3 ten Stelle. Man sieht daraus z. B., dass die Correction der Säcularianderungen in AR. im Maximo, d. i. für $18^{\text {h }}$ lrei nördl. und für $6^{\text {h }}$ bei sildlichen Declinationen

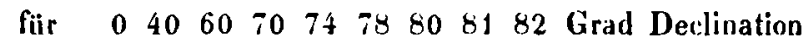
beträgt $+21 \quad 26 \quad 32 \quad 3843 \quad 51 \quad 57 \quad 6167$ Eiulseiten d. 4. Stelle.

Sobald aber die eigene Bewegung des Sterns merklich wird, ist, besonders für hohe Declinationen, die Genauigkeit bis anf eine Einheit resp. der $4^{\text {ten }}$ und $3^{\text {ten }}$ Stelle nur illusorisch, indem der zweite und die folgenden Differentialquotienten Glieder entbalten, die von der eigenen Bewegung abhängig sind, und doch noch zur reinen Reduction gehören, so dass der erst wach ihrer Anbringung sich noch zeigende Unterschied der Wirkung der eigenen Bewegung allein zugeschrieben werden kann. Statt nämlich $p$ und $p^{\prime}$ für $d \alpha$ und $d \delta$ zu setzen, hätten wir $p+\mu$ und $p^{\prime}+\mu^{\prime}$ nchmen müssen, wenn $\mu$ und $\mu$ die eigenen Bewegungeu in AR. und Decl. bezeichnen. Dadurch ist dem $d p$ hinzuzufügen $\mu p^{\prime} \operatorname{tg} d \sin 1^{n}$ $+\mu^{\prime} n \sec \delta^{2} \sin \alpha \sin 1^{\prime \prime}$ und dem $d p^{\prime} n o c h-\mu n \sin \alpha \sin 1^{\prime \prime}$. (S. Tab. Reg. p. XIV. und Argelander: Positiones mediae p. XII.) Das Verfahren, zur Ableitung der Säcularänderungen sich aweier Präcessionen zu bedienen, die auf zu ver- 